\title{
Magnetic In-Vacuum Field Mapping System for the Muon g-2 Experiment
}

\author{
Peter Winter** \\ High Energy Physics Division \\ Argonne National Laboratory E-mail: winterp@an 1 . gov
}

\begin{abstract}
The Muon g-2 experiment at Fermilab will measure the anomalous magnetic moment of the muon, $a_{\mu}$ with a precision of $140 \mathrm{ppb}$, a four-fold improvement over the former BNL E821 result. If the current discrepancy of about $3.5 \sigma$ between the Standard Model value for $a_{\mu}$ and the former experiments was real, then the new experiment would verify the deviation with $>7 \sigma$ significance. The experiment uses muons with the magic momentum of $3.09 \mathrm{GeV} / \mathrm{c}$ stored in a very homogeneous magnetic field of a $45 \mathrm{~m}$ long storage ring. The determination of $a_{\mu}$ requires the precise knowledge of both the muon spin precession, $\omega_{a}$, and the magnetic field, $\omega_{p}$, expressed in terms of the free-proton Larmor frequency. The field measurement uses the high precision tool of pulsed proton Nuclear Magnetic Resonance and requires cross-calibrating a set of probes: a very accurate NMR probe calibrates the 17 probes on the in-vacuum field mapping system (the so-called trolley). This trolley in turn is used to map the field over the full azimuth of the storage ring. The trolley measurement also provides the calibration of the 378 fixed probes around the ring that constantly monitor the field drift. The trolley is particularly important as it measures the relevant field that is seen by the stored muons and this article presents the details of this field mapping system. This work was supported in part by the US DOE, Fermilab and US DOE OHEP under contract No. KA2201020.
\end{abstract}

ICHEP 2018, International Conference on High Energy Physics

4-11 July 2018

Seoul, South Korea

\footnotetext{
*Speaker.

${ }^{\dagger}$ for the Muon g-2 Collaboration
} 


\section{The In-Vacuum Magnetic Field Mapping System}

The measurement of the muon anomalous magnetic moment requires the measurement of the anomalous spin precession frequency, $\omega_{a}$, as well as the precise knowledge of the magnetic field in terms of the free proton Larmor frequency, $\omega_{p}$. Determination of $\omega_{a}$ is based on the time and energy spectrum of the decay positrons and is described elsewhere in this proceedings. The measurement of the magnetic field with a systematic uncertainty of better than $70 \mathrm{ppb}$ (due to the very high precision of the pNMR technique the statistical uncertainty is negligible) is achieved through the following main ingredients: 1) Passive and active shimming to prepare a very uniform field, 2) constant monitoring of the storage field drift over time via 378 NMR probes mounted above and below the storage region, 3) frequent field mapping of the storage region with an in-vacuum trolley system, and 4) the absolute calibration with a very accurate and precise water-based NMR probe. The in-vacuum field mapping trolley plays an essential role in measuring the magnetic field at the muon storage region. The left panel of Fig. 1 shows the dipole field measured by the trolley.

The trolley system was inherited from the BNL E821 experiment and underwent significant upgrades. The on-board, obsolete controller was completely replaced with a low-power SmartFusion processor that facilitates the control of the NMR measurement sequences, the data readout and communication with the interface outside the vacuum. The development of this new board was challenged by the limitation of a single coaxial cable that must provide the power, a $62 \mathrm{MHz}$ precise reference clock signal, and the data communication. Two new key upgrades facilitated to achieve the single shot precision of better than $1 \mathrm{ppb}$ : the NMR FID signal is fully digitized on-board with a 16-bit, 1 MSPS ADC and 2) RF switches on-board and in the interface module separate the $62 \mathrm{MHz}$ clock from the data communication. Improvements in the azimuthal position determination of the trolley will come through the addition of a barcode reader board that records regular and irregular marks on the bottom of the vacuum chambers (see the right panel of Fig. 1).

The mechanism to park the trolley during muon injection and the cable pulling drums were refurbished from E821. Both systems are operated by piezo-electric motors that can operate in the high magnetic field. Their motion control was replaced by a commercial Galil controller combined with custom built electronics interfacing with the motors, tension sensors, rotary encoders, and other sensors. Both the new trolley NMR electronics and motion control system were successfully commissioned and have been operating well during the first data taking run of the experiment.
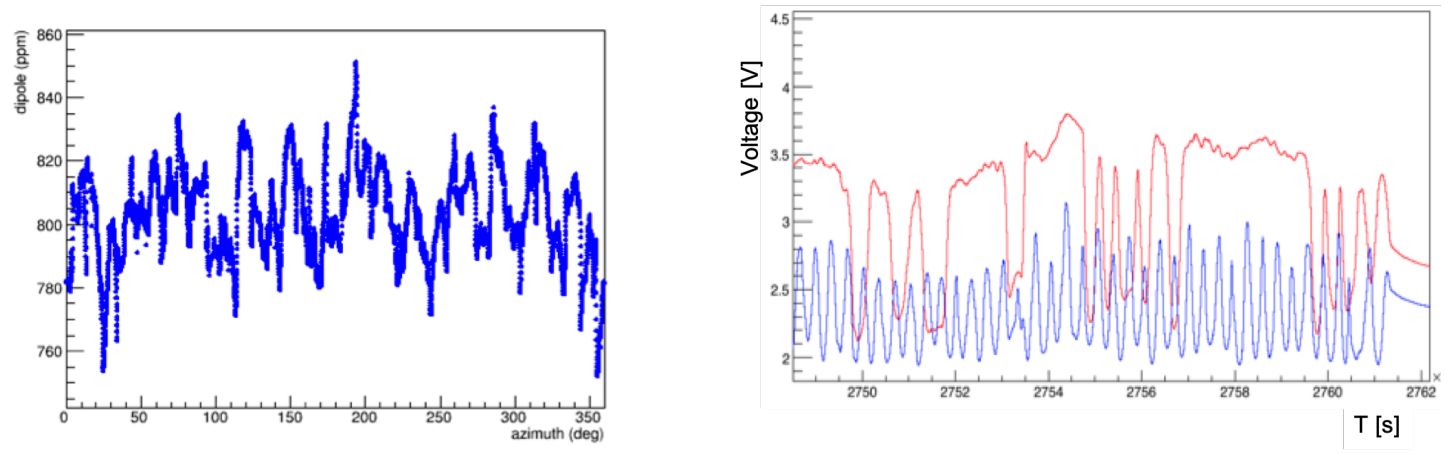

Figure 1: Left panel: Dipole field measured with the new in-vacuum field mapping system. Right panel: Digitized regular barcode marks (blue) and irregular patterns (red). 